\title{
An economical system incorporating obsolete computers in an operant conditioning laboratory
}

\author{
DAVID M. SARGENT \\ State University of New York at Oswego, Oswego, New York
}

\begin{abstract}
A variety of obsolete computers were used with a commercial interface device (Alpha Bus) to control animal (rat) operant chambers. Original 28VDC electromechanical equipment was also used in conjunction with the interface to reduce initial equipment costs and maintain broad com. patibility. Effective response counting and event control in three separate operant chambers was achieved through the use of secondary processing available on the current interface device. Advantages of the present systems include low initial and maintenance costs, expandability, and adaptability to different computers and applications.
\end{abstract}

Several laboratories have used specific low-cost computers and peripheral equipment to assemble operant laboratories. These low-cost computer operant systems have involved the TRS-80 (O'Dell, 1983), the Commodore-64 (Jackson \& O'Dell, 1985; O'Dell \& Jackson, 1986; Wynne, 1990), the NorthStar and other S-100 systems (Sargent, 1989), the Apple II series (Scandrett \& Gormezano, 1980), the IBM PC and compatibles (Lavond \& Steinmetz, 1989), and the Apple Macintosh (Blumenthal \& Cooper, 1990). While each of these systems is ideally suited for certain experiments and applications, each is somewhat customized and the interface or peripheral equipment is specific to a given type of computer. The present systems involve a commercial interface device that is easily adaptable to a wide variety of the older, obsolete computers. While each computer has its own idiosyncrasies and operating procedures, there are common programming languages, such as BASIC and even Forth, that can be used across computers with only minor changes. Given that it is sometimes questionable why anyone would want a potpourri of computers populating a laboratory, there are, however, many advantages, such as increased equipment availability, expandability, and incumbent breadth of knowledge (if the laboratory is also used for teaching students that will eventually move on to systems of unknown origin), across different computer systems.

Very often, academic departments and individuals will update their computer systems with newer state-of-theart products and are willing to sell their old systems for a fraction of the original cost. I have acquired several KayPro CP/M computers, TRS-80s, Apple Iles and II +s, and a variety of IBM PC-compatibles, enough to equip a small teaching laboratory. Using these economical, but obsolete, machines poses certain obstacles, such as com-

Correspondence should be addressed to David M. Sargent, Department of Psychology, SUNY at Oswego, Oswego, NY 13126. patible interfacing equipment and limited processor cycling speed. The standard for interfacing equipment in the past has been the IBM bus, making only certain IBM PCtype machines compatible for interfacing. Several manufacturers are now offering low-cost interfaces that connect to a standard serial port. I am currently using a system from Alpha Products, which, in addition to a serial connection, can be easily adapted to the IBM bus, Apple bus, the Tandy TRS-80 connector, or even the Commodore64 user port. This particular feature allows a variety of different computers to work similarly within the same laboratory.

\section{AIMS OF THE PRESENT SYSTEMS}

Cycle speed is generally not a problem if the computer is only responsible for output signals. Most computers can easily present a variety of tones, lights, and other stimuli with processor speeds as low as $2.2 \mathrm{MHz}$. Problems arise when the processor does not cycle fast enough to record every switch closure response, especially in the case of multiple inputs (the present system has one computer controlling three operant chambers). The Alpha Bus interface allows for the use of a secondary processor, a feature also offered by some other manufacturers. With the secondary processor dedicated to recording inputs, the host processor can control experimental events, time intervals, and display cumulative responses through program calls to the secondary processors. To control experimental sessions, the present system uses a BASIC or a Forth programming language that calls responses from the secondary processor: either a dedicated counting register or a dedicated 8052 processor on the Alpha Bus interface. Barpress switch closures are counted along with photodiode monitors in the food magazine (registering exact time of pellet acquisition by the subject). This utilization of a secondary processor allows for great flexibility in terms of both host processor and types of input. For 


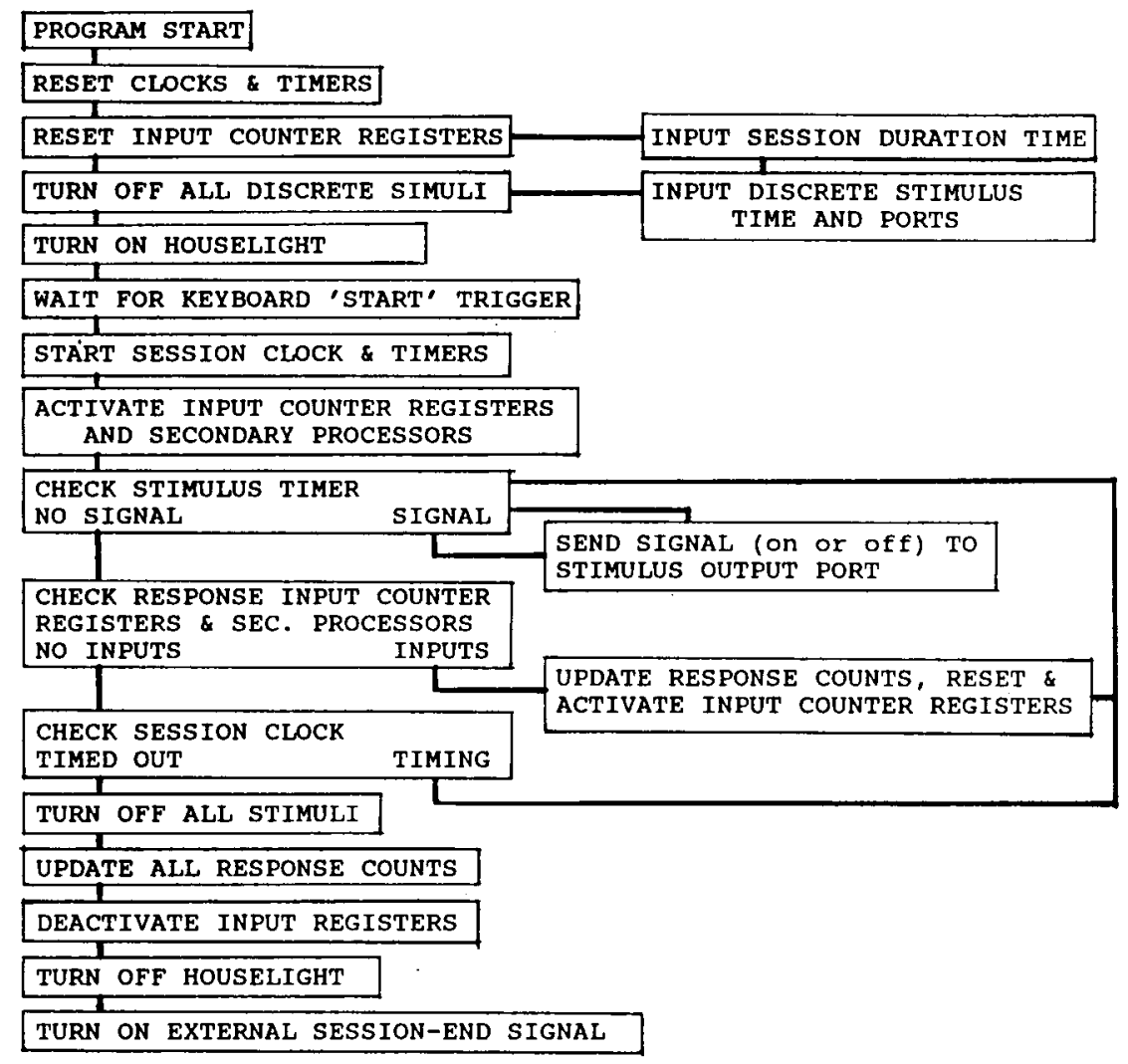

Figure 1. A top-down flow chart utilizing modular stages will work with a variety of programming languages. Vertical lines connecting the stages on the left indicate top-down sequential operation. Connection lines on the right indicate loops.

example, additions to the present systems will include photodiode position sensors in the chamber itself and realtime temperature readings from the subject during the session. In connecting any computer system to a real-time environment, the primary concern is to simplify not only the necessary hardware, but also the operation of the equipment. This often results in a direct tradeoff; a quick hookup usually requires a more complicated "use" procedure and a system that has a "bulletproof" operation takes extensive setup and testing. Usually a system will sacrifice some ease of operation (function) for some flexibility in application. In these days of equipment justification, it is almost necessary to have a wide variety of possible applications for a piece of equipment.

In using low-speed, low-memory capacity computers to record rapid real-time events the system must either be dedicated to the sampling of the events, or a temporary event storage system must be employed. If there is a possibility of more than one event per channel occurring within a single clock cycle, the system will "miss" the event. The solution is to store the events in registers that can be read, verified, and cleared within either a clock or a program cycle. For example, the Alpha Bus system has a counter/timer that utilizes three 16-bit registers to store switch closure events. By using these registers, the slow Z-80A, 8502 , or 8088 can involve other routines within a program cycle and not risk missing any of the counts that are occurring. Figure 1 shows a generalized flow chart of a BASIC program that will run a wide variety of conditioning sessions. Forth programs utilize the same flow chart with programmed :words (colon definitions) for each of the stages, greatly simplifying program variations.

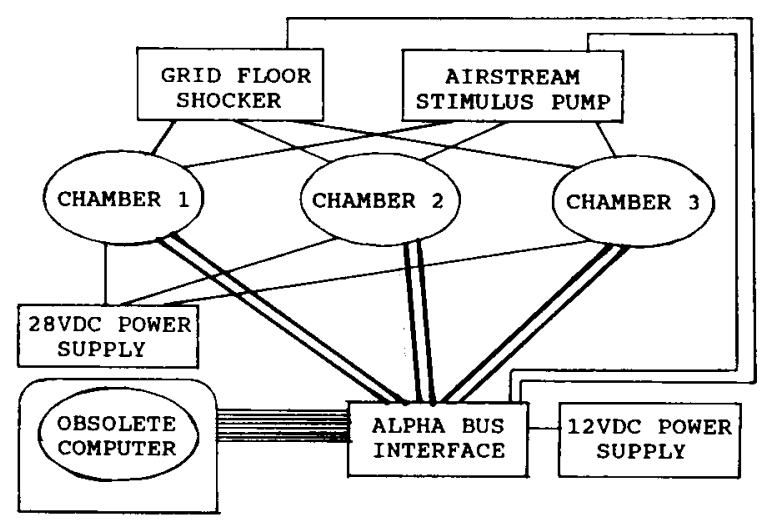

Figure 2. A schematic of an experimental station capable of running three chambers concurrently is shown. This configuration maximizes the chamber to peripheral equipment ratio. 
The programming complexity of setting, resetting, reading, data dumping, and clearing the counting registers can be a problem in terms of flexibility. In our laboratory, complex contingencies are often set up requiring switch counts combined with timed intervals resulting in specific outcomes. Program routines to handle these contingencies are not always feasible when contained in the primary programming loop; the program itself becomes unwieldy. At this point, secondary processing beyond the utilization of mere counting registers is beneficial. Secondary processors, such as the serial interface processor produced by Alpha Bus, can be programmed to run independently, thereby controlling outcome contingencies. The primary processor can operate running "unaware" of the secondary processor until such time as an event or signal is needed. While this hierarchical system appears to be quite complex on the surface, each individual subsystem is quite simple and easy to program and debug. Secondary processor programs can be prewritten, thereby making the operation of the system very simple to check and use. The demands of real-time programming are not always best handled by throwing in bigger and faster processors.

\section{INTERFACE CONNECTIONS AND CONTROL}

The present systems use a 28VDC power supply, response lever, food magazine, pulse former, houselights, cue lights, and so forth from the original Lehigh Valley, Gerbrands, and Grason-Stadtler equipment (modifications were easily made to also include the 12VDC Coulbourn equipment). The interface itself requires a $12 \mathrm{VDC}$ power supply that eliminates the need for a separate supply to switch the Coulbourn floor shocker and Radio Shack buzzers (tone). An additional relay was used to switch the 120VAC air pump used for the airstream tactile stimulus. Figure 2 shows the rough layout of the experimental station with the major components.

The barpress levers were connected directly to the 28VDC power supply and the pulse formers (used here without the traditional power rods). The pulse-former output was then connected to both the interface input secondary processor and the output relay, as seen in Figure 3. The use of 28VDC pulse formers reduces the amount of switch debounce programming often performed by the host processor. Operant reinforcement, contingent on the specific barpress, is done merely by operating the appropriate relay(s) without having to time either the signal duration or the onset to the food magazine.

Connecting three operant chambers to a single computer and interface was considered optimal for several reasons. The reduction in the amount of equipment needed to simultaneously run 3 experimental subjects was balanced with the flexibility of being able to stagger session start times or session types. The present laboratory has two stations (see Figure 2) with three chambers each, allowing, for example, the simultaneous running of 3 subjects on continuous reinforcement and three on a variable interval schedule, with four discrete presentations of airstream and tone followed by shock. Additionally, the Alpha Bus counter/timer has three 16-bit registers, allowing one to be dedicated to each chamber. Since standard operant conditioning paradigms usually require only individual subject contingencies for barpress reinforcement, discrete events such as tone, cue light, houselight, airstream, and shock can be presented to all three chambers simultaneously with a single relay. The present laboratory uses all of these stimuli switched by the remaining five relays on the interface output card, as shown in

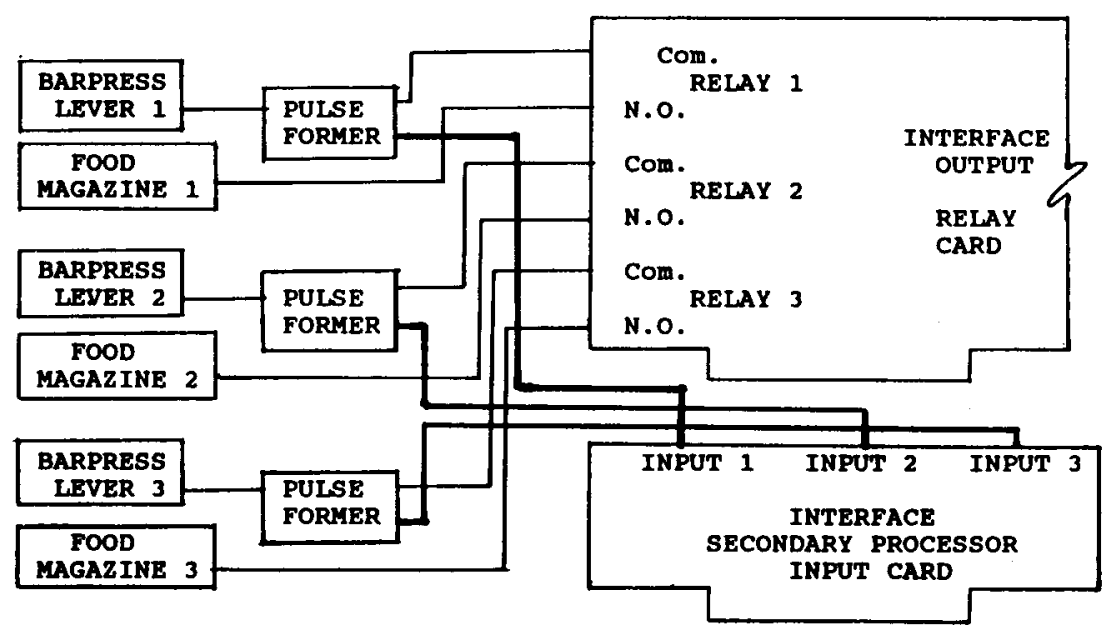

Figure 3. The use of $28 \mathrm{VDC}$ manipulanda and pulse formers reduces the signal processing burden of the host computer and the secondary processor. 


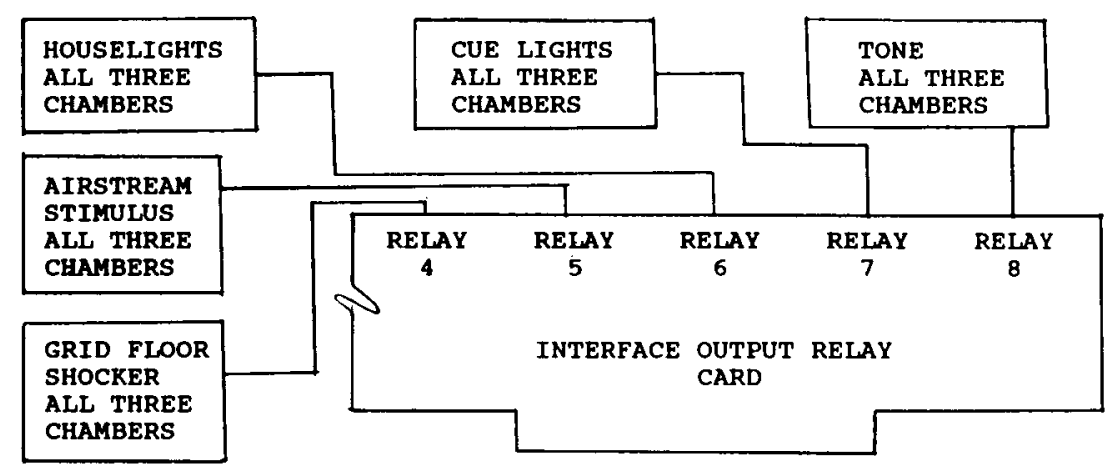

Figure 4. Presentation of discrete stimulus events are yolked across all three chambers, effectively reducing processor load and the number of needed peripherals.

Figure 4. If more outputs are needed for a given station, either a second output card can be added to the Alpha Bus, or a simple binary decoder can be attached, thereby multiplying the number of available outputs.

\section{FUTURE EXPANSION}

All of the signals discussed in this paper were digital. Future expansion will include a thermistor (thermal resistor) temperature sensor in each operant chamber to measure the breathing rate of the subjects while they are at the barpress manipulanda. These data will require analog-todigital (A/D) conversion, which is available on a card from Alpha Products. Given the wide variety of signal capabilities, response measurement and event control are virtually limitless in this application. Future equipment updates and expansion are also possible with the present stations, which allow upward compatibility to 80286,80386 , and even 80486 processor systems. Alpha Products is also, as of this writing, developing an adapter for the Nu-Bus connector present in the Apple Macintosh II series computers.

\section{REFERENCES}

Blumenthal, T. D., Cooper, J. A. (1990). Using the Macintosh computer in psychophysical research: Programs for stimulus presentation, data collection, and response quantification. Behavioral Research Methods, Instruments, \& Computers, 22, 99-104.

JACKSON, D. E., O'DELL, J. W. (1985). The VIC-20 computer and an interface system for controlling operant chambers. Behavioral Research Methods, Instruments, \& Computers, 17, 406-408.

Lavond, D. G., SteinmetZ, J. E. (1989). An inexpensive interface for the IBM PC/XT and compatibles. Behavioral Research Methods, Instruments, \& Computers, 21, 435-440.

O'DELL, J. W. (1983). TRS-80 as a controller: An introduction to interfacing. Peterborough, NH: Green Books.

O'DelL, J. W., \& JCKSON, D. E. (1986). The Commodore-64 and an interface system for controlling operant chambers. Behavioral Research Methods, Instruments, \& Computers, 18, 339-341.

SARGENT, D. M. (1989). Use of on-line computers in experimental psychology: Hardware considerations. Behavioral Research Methods, Instruments, \& Computers, 21, 163-165.

SCANDRett, J., \& Gormezano, I. (1980). Microprocessor control and A/D data acquisition in classical conditioning. Behavioral Research Methods, Instruments, \& Computers, 12, 120-125.

WyNNE, C. D. L. (1990). A Commodore 64-based interfaced system for the operant laboratory. Behavioral Research Methods, Instruments, \& Computers, 22, 27-33. 